\title{
On a singular perturbed problem in an annulus
}

\author{
SANJIBAN SANTRA AND JUNCHENG WEI
}

\begin{abstract}
In this paper we prove the conjecture due to Ruf-Srikanth [14]. We prove the existence of positive solution under Dirichlet and Neumann boundary conditions, which concentrate near the inner boundary and outer boundary of an annulus respectively as $\varepsilon \rightarrow 0$. In fact, our result is independent of the dimension of $\mathbb{R}^{N}$.
\end{abstract}

Mathematics Subject Classification (2010): 35B08 (primary); 35J20, 35J65 (secondary).

\section{Introduction}

There has been a considerable interest in understanding the behavior of positive solutions of the elliptic problem

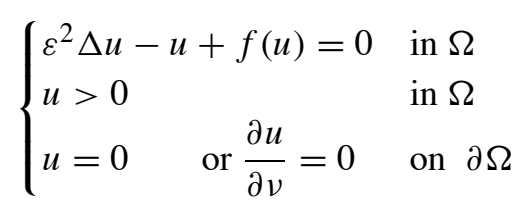

where $\varepsilon>0$ is a parameter, $f$ is a superlinear nonlinearity and $\Omega$ is a smooth bounded domain in $\mathbb{R}^{N}$. Let $F(u)=\int_{0}^{u} f(t) d t$. We consider the problem when $f(0)=0$ and $f^{\prime}(0)=0$. This type of equations arise in various mathematical models derived from population theory, chemical reactor theory see Gidas-NiNirenberg [6]. In the Dirichlet case, $\mathrm{Ni}$ - Wei showed in [19] that the least energy solutions of equation (1.1) concentrate, for $\varepsilon \rightarrow 0$, to single peak solutions, whose maximum points $P_{\varepsilon}$ converge to a point $P$ with maximal distance from the boundary $\partial \Omega$. In the Neumann case, Ni-Takagi [17] showed that for sufficiently small $\varepsilon>0$, the least energy solution is a single boundary spike and has only one local maximum $P_{\varepsilon} \in \partial \Omega$. Moreover, in [18], they prove that $H\left(P_{\varepsilon}\right) \rightarrow \max _{P \in \partial \Omega} H(P)$ as $\varepsilon \rightarrow 0$ where $H(P)$ is the mean curvature of $\partial \Omega$ at $P$. A simplified proof was

Received October 17, 2013; accepted in revised form September 20, 2014.

Published online February 2016. 
given by del Pino-Felmer in [3], for a wider class of nonlinearities using a method of symmetrization.

We mention some nice results on the multiple boundary and interior peaked solutions for the Neumann case of (1.1). For the single and multiple boundary spikes, Gui [8] constructed multiple boundary spike solutions at multiple local maximum points of $H(P)$, using a variational method. Wei [21] and Wei-Winter [22] constructed single and multiple boundary spike solutions at multiple non-degenerate critical points of $H(P)$, using the Lyapunov-Schmidt reduction method. Later on Y.Y.Li [10] and del Pino-Felmer-Wei [4] constructed single and multiple boundary spikes in the degenerate case. For a detailed bibliography on this topic, we refer to the review article by $\mathrm{Ni}[16]$.

Higher dimensional concentrating solutions was studied by Ambrosetti-Malchiodi-Ni in $[1,2]$; they consider solutions which concentrate on spheres, i.e. on $(N-1)$ - dimensional manifolds. They studied the problem

$$
\begin{cases}\varepsilon^{2} \Delta u-V(r) u+f(u)=0 & \text { in } A \\ u>0 & \text { in } A \\ u=0 & \text { on } \partial A\end{cases}
$$

in an annulus $A=\left\{x \in \mathbb{R}^{N}: 0<a<|x|<b\right\}, V(r)$ is a smooth radial potential bounded below by a positive constant. They introduced a modified potential $M(r)=$ $r^{N-1} V^{\theta}(r)$, with $\theta=\frac{p+1}{p-1}-\frac{1}{2}$, satisfying $M^{\prime}(b)<0$ (respectively $M^{\prime}(a)>0$ ), then there exists a family of radial solutions which concentrates on $|x|=r_{\varepsilon}$ with $r_{\varepsilon} \rightarrow b$ (respectively $r_{\varepsilon} \rightarrow a$ ) as $\varepsilon \rightarrow 0$. In fact, they conjectured that in $N \geq 3$ there could exist also solutions concentrating to some manifolds of dimension $k$ with $1 \leq k \leq N-2$. Moreover, in $\mathbb{R}^{2}$, concentration of positive solutions on curves in the general case was proved by del Pino-Kowalczyk-Wei [5]. The Neumann case was studied by Malchiodi-Montenegro [11,12].

In Esposito et al. [7], the asymptotic behavior of radial solutions for the singularly perturbed elliptic problem (1.2) was studied using the Morse index information to describe the complete description of the blow-up behavior. As a result, they exhibit sufficient conditions which guarantee that radial ground state solutions blow-up and concentrate at the inner or outer boundary of the annulus. For more, interesting consequences, see Pacella-Srikanth [13] and Ruf-Srikanth [14,15].

In this paper, we consider the following two singular perturbed problems,

$$
\begin{aligned}
& \begin{cases}\varepsilon^{2} \Delta u-u+u^{p}=0 & \text { in } A \\
u>0 & \text { in } A \\
u=0 & \text { on } \partial A\end{cases} \\
& \begin{cases}\varepsilon^{2} \Delta u-u+u^{p}=0 & \text { in } A \\
u>0 & \text { in } A \\
\frac{\partial u}{\partial v}=0 & \text { on } \partial A\end{cases}
\end{aligned}
$$


where $A$ is an annulus in $\mathbb{R}^{N}=\mathbb{R}^{M} \times \mathbb{R}^{K}$ with $A=\left\{x \in \mathbb{R}^{N}: 0<a<\right.$ $|x|<b\}, \varepsilon>0$ is a small number and $v$ denotes the unit normal to $\partial A$ and $N \geq 2$. In this paper, we are interested in finding solution $u(x)=u(r, s)$ where $r=\sqrt{x_{1}^{2}+x_{2}^{2}+\cdots x_{M}^{2}}$ and $s=\sqrt{x_{M+1}^{2}+x_{M+2}^{2}+\cdots x_{K}^{2}}$.

Let us consider the conjecture due to Ruf and Srikanth:

Does there exist a solution for the problems (1.3) and (1.4), which concentrates on $\mathbb{R}^{M+K-1}$ dimensional subsets as $\varepsilon \rightarrow 0$ ?

Theorem 1.1. For $\varepsilon>0$ sufficiently small, there exists a solution of (1.3) which concentrates near the inner boundary of $A$.

Theorem 1.2. For $\varepsilon>0$ sufficiently small, there exists a solution of (1.4) which concentrates near the outer boundary of $A$.

\section{Set up for the approximation}

Note that, under symmetry assumptions, $A$ can be reduced to a subset of $\mathbb{R}^{2}$ where $\mathcal{D}=\left\{(r, s): r>0, s>0, a^{2}<r^{2}+s^{2}<b^{2}\right\}$. Let $P_{\varepsilon}=\left(P_{1, \varepsilon}, P_{2, \varepsilon}\right)$ be a point of maximum of $u_{\varepsilon}$ in $A$, then $u_{\varepsilon}\left(P_{\varepsilon}\right) \geq 1$. From (1.3) we obtain

$$
\varepsilon^{2} u_{r r}+\varepsilon^{2} u_{s s}+\varepsilon^{2} \frac{(M-1)}{r} u_{r}+\varepsilon^{2} \frac{(K-1)}{s} u_{s}-u+u^{p}=0
$$

Let $\mathcal{D}_{1}, \mathcal{D}_{2}$ are the inner and outer boundary of $\mathcal{D}$ respectively and $\mathcal{D}_{3}, \mathcal{D}_{4}$ are the horizontal and vertical boundary of $\mathcal{D}$ respectively.

If $P=\left(P_{1}, P_{2}\right)$ be a point in $\mathcal{D}$ such that $\operatorname{dist}\left(P, \mathcal{D}_{1}\right)=d$, then we can express

$$
P_{1}=(a+d) \cos \theta ; P_{2}=(a+d) \sin \theta
$$

where $\theta$ is the angle between the $x$-axis and the line joining $P$. Furthermore, if $\operatorname{dist}\left(P, \mathcal{D}_{2}\right)=d$, then we can express

$$
P_{1}=(b-d) \cos \theta ; P_{2}=(b-d) \sin \theta .
$$

See Figure 2.1 and Figure 2.2.

The functional associated to the problem is

$$
I_{\varepsilon}(u)=\int_{\mathcal{D}} r^{M-1} s^{K-1}\left(\frac{\varepsilon^{2}}{2}|\nabla u|^{2}+\frac{1}{2} u^{2}-\frac{1}{p+1} u^{p+1}\right) d r d s .
$$

Moreover, (1.3) reduces to

$$
\begin{cases}\varepsilon^{2} u_{r r}+\varepsilon^{2} u_{s s}+\varepsilon^{2} \frac{(M-1)}{r} u_{r}+\varepsilon^{2} \frac{(K-1)}{s} u_{s}-u+u^{p}=0 & \text { in } \mathcal{D} \\ u=0 & \text { on } \mathcal{D}_{1} \cup \mathcal{D}_{2} \\ \frac{\partial u}{\partial v}=0 & \text { on } \mathcal{D}_{3} \cup \mathcal{D}_{4} .\end{cases}
$$




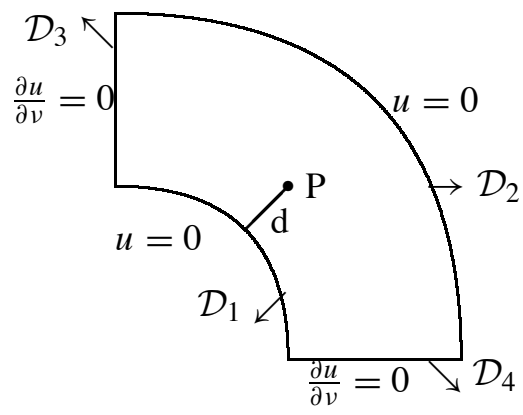

Figure 2.1. Dirichlet case

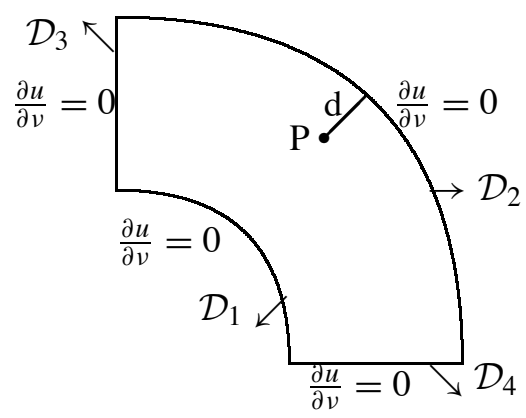

Figure 2.2. Neumann Case

Re-scaling about the point $P$, we obtain in $A_{\varepsilon}$

$$
u_{r r}+u_{s s}+\varepsilon \frac{(M-1)}{P_{1}+\varepsilon r} u_{r}+\varepsilon \frac{(K-1)}{P_{2}+\varepsilon s} u_{s}-u+u^{p}=0 .
$$

The entire solution associated to (2.1) where $U$ satisfies

$$
\begin{cases}\Delta_{(r, s)} U-U+U^{p}=0 & \text { in } \mathbb{R}^{2} \\ U(r, s)>0 & \text { in } \mathbb{R}^{2} \\ U(r, s) \rightarrow 0 & \text { as }|(r, s)| \rightarrow \infty\end{cases}
$$

Moreover $U$ is non-degenerate, which means

$$
\operatorname{Ker}\left[\Delta_{(r, s)}-1+p U^{p-1}\right]=\left\{\frac{\partial U}{\partial r}, \frac{\partial U}{\partial s}\right\}
$$

Let $z=(r, s)$. Moreover, $U(z)=U(|z|)$ and the asymptotic behavior of $U$ at infinity is given by

$$
\left\{\begin{array}{l}
U(z)=A|z|^{-\frac{1}{2}} e^{-|z|}\left(1+O\left(\frac{1}{|z|}\right)\right) \\
U^{\prime}(z)=-A|z|^{-\frac{1}{2}} e^{-|z|}\left(1+O\left(\frac{1}{|z|}\right)\right)
\end{array}\right.
$$

for some constant $A>0$.

Let $K(z)$ denote the fundamental solution of $-\Delta_{(r, s)}+1$ centered at 0 . Then, for $|z| \geq 1$, we have

$$
\left\{\begin{array}{l}
U(z)=\left(B+O\left(\frac{1}{|z|}\right)\right) K(z) \\
U^{\prime}(z)=\left(-B+O\left(\frac{1}{|z|}\right)\right) K(z)
\end{array}\right.
$$

for some positive constant $B$. 
Let $U_{\varepsilon, P}(z)=U\left(\left|\frac{z-P}{\varepsilon}\right|\right)$. Now we construct the projection map for the Dirichlet case as

$$
\begin{cases}\varepsilon^{2} \Delta_{(r, s)} P U_{\varepsilon, P}-P U_{\varepsilon, P}+U_{\varepsilon, P}^{p}=0 & \text { in } \mathcal{D} \\ P U_{\varepsilon, P}(r, s)>0 & \text { in } \mathcal{D} \\ P U_{\varepsilon, P}(r, s)=0 & \text { on } \partial \mathcal{D},\end{cases}
$$

and the projection in the Neumann case as

$$
\begin{cases}\varepsilon^{2} \Delta_{(r, s)} Q U_{\varepsilon, P}-Q U_{\varepsilon P}+U_{\varepsilon, P}^{p}=0 & \text { in } \mathcal{D} \\ Q U_{\varepsilon, P}(r, s)>0 & \text { in } \mathcal{D} \\ \frac{Q U_{\varepsilon, P}}{\partial \nu}(r, s)=0 & \text { on } \partial \mathcal{D} .\end{cases}
$$

If $v_{\varepsilon}=U_{\varepsilon, P}-P U_{\varepsilon, P}$ and $w_{\varepsilon}=U_{\varepsilon, P}-Q U_{\varepsilon, P}$, then we have

$$
\begin{gathered}
\begin{cases}\varepsilon^{2} \Delta_{(r, s)} v_{\varepsilon}-v_{\varepsilon}=0 & \text { in } \mathcal{D} \\
v_{\varepsilon}=U_{\varepsilon, P} & \text { on } \partial \mathcal{D},\end{cases} \\
\begin{cases}\varepsilon^{2} \Delta_{(r, s)} w_{\varepsilon}-w_{\varepsilon}=0 & \text { in } \mathcal{D} \\
\frac{\partial w_{\varepsilon}}{\partial v}=\frac{\partial U_{\varepsilon, P}}{\partial v} & \text { on } \partial \mathcal{D} .\end{cases}
\end{gathered}
$$

Consider the function $s(\theta)=\cos ^{M-1} \theta \sin ^{K-1} \theta$ in $\left[0, \frac{\pi}{2}\right]$. Then neither $\theta_{0}=0$ nor $\theta_{0}=\frac{\pi}{2}$ are points of maxima of $s$. But $s>0$ and hence $\theta_{0}$ lies in $\left(0, \frac{\pi}{2}\right)$.

Furthermore, consider the function $h(d)=d+e^{-\frac{d}{\varepsilon}}$ in $0<d<1$. Then $h$ attains its minimum at a point $d=\varepsilon|\ln \varepsilon|$.

For any $\theta \in\left[\theta_{0}-\delta, \theta_{0}+\delta\right]$; we define the configuration space for the Dirichlet and Neumann case as

$$
\Lambda_{\varepsilon, D}=\left\{P \in \mathcal{D}: \operatorname{dist}\left(P, \mathcal{D}_{1}\right) \geq \frac{k}{2} \varepsilon \ln \frac{1}{\varepsilon}\right\}
$$

and

$$
\Lambda_{\varepsilon, N}=\left\{P \in \mathcal{D}: \operatorname{dist}\left(P, \mathcal{D}_{2}\right) \geq \frac{k}{2} \varepsilon \ln \frac{1}{\varepsilon}\right\}
$$

respectively for some $k>0$ small.

We develop the following lemma similar to Lin, Ni and Wei [9].

Lemma 2.1. Assuming that $\frac{k}{2} \varepsilon|\ln \varepsilon| \leq d\left(P, \mathcal{D}_{1}\right) \leq \delta$, then we obtain

$$
v_{\varepsilon}(z)=(B+o(1)) K\left(\frac{\left|z-P^{\star}\right|}{\varepsilon}\right)+O\left(\varepsilon^{2+\sigma}\right),
$$

where $P^{\star}=P+2 d\left(P, \mathcal{D}_{1}\right) \nu_{\bar{P}}$ and $\bar{P} \in \mathcal{D}_{1}$ is a unique point such that $d(P, \bar{P})=$ $2 d\left(P, \mathcal{D}_{1}\right)$, while $\sigma$ is a small positive number; $\delta$ is sufficiently small. Moreover, $\nu_{\bar{P}}$ is the outer unit normal at $\bar{P}$. 
Proof. Define

$$
\begin{cases}\varepsilon^{2} \Delta_{(r, s)} \Psi_{\varepsilon}-\Psi_{\varepsilon}=0 & \text { in } \mathcal{D} \\ \Psi_{\varepsilon}>0 & \text { in } \mathcal{D} \\ \Psi_{\varepsilon}=1 & \text { on } \partial \mathcal{D} .\end{cases}
$$

Then for sufficiently small $\varepsilon, \Psi_{\varepsilon}$ is uniformly bounded.

But for $z \in \partial \mathcal{D}$, we obtain

$$
U_{\varepsilon, P}(z)=U\left(\frac{|z-P|}{\varepsilon}\right)=(A+o(1)) \varepsilon^{\frac{1}{2}}|z-P|^{-\frac{1}{2}} e^{-\frac{|z-P|}{\varepsilon}} .
$$

First, we have

$$
U_{\varepsilon, P}(z)=(B+o(1)) K\left(\frac{|z-P|}{\varepsilon}\right) .
$$

Hence by the comparison principle we obtain, for some $\sigma>0$ small,

$$
v_{\varepsilon} \leq C \varepsilon^{2+\sigma} \Psi_{\varepsilon} \text { whenever } d\left(P, \mathcal{D}_{1}\right) \geq 2 \varepsilon|\ln \varepsilon| .
$$

Therefore, it remains to check whether (2.16) holds in

$$
\frac{k}{2} \varepsilon|\ln \varepsilon| \leq d\left(P, \mathcal{D}_{1}\right) \leq 2 \varepsilon|\ln \varepsilon| \text {. }
$$

Define the function

$$
\phi_{1}(z)=\left(B-\varepsilon^{\frac{1}{4}}\right) K\left(\frac{\left|z-P^{\star}\right|}{\varepsilon}\right)+\varepsilon^{2+\sigma} \Psi_{\varepsilon} .
$$

Then $\phi_{1}$ satisfies

$$
\varepsilon^{2} \Delta_{(r, s)} \phi_{1}-\phi_{1}=0 .
$$

For any $z$ in $\mathcal{D}_{1}$ with $|z-P| \leq \varepsilon^{\frac{3}{4}}$ we have

$$
\frac{|z-P|}{\varepsilon}=\left(1+O\left(\varepsilon^{\frac{1}{2}}\right)|\ln \varepsilon|\right) \frac{\left|z-P^{\star}\right|}{\varepsilon}
$$

and hence

$$
v_{\varepsilon} \leq \phi_{1} .
$$

For any $z \in \mathcal{D}_{1}$ with $|z-P| \geq \varepsilon^{\frac{3}{4}}$ we have

$$
v_{\varepsilon}(z) \leq C e^{-\varepsilon^{-\frac{1}{4}}} \leq \varepsilon^{2+\sigma} \leq \phi_{1} .
$$

Summarizing, we obtain,

$$
v_{\varepsilon} \leq \phi_{1} \text { for all } z \in \mathcal{D}_{1} .
$$

Similarly, we obtain the lower bound for $z \in \mathcal{D}_{1}$,

$$
v_{\varepsilon}(z) \geq\left(B+\varepsilon^{\frac{1}{4}}\right) K\left(\frac{\left|z-P^{\star}\right|}{\varepsilon}\right)-\varepsilon^{2+\sigma} \Psi_{\varepsilon} .
$$


Corollary 2.2. Assume that $\frac{k}{2} \varepsilon|\ln \varepsilon| \leq d\left(P, \mathcal{D}_{2}\right) \leq \delta$ where $\delta$ is sufficiently small. Then

$$
w_{\varepsilon}(z)=-(B+o(1)) K\left(\frac{\left|z-P^{\star}\right|}{\varepsilon}\right)+O\left(\varepsilon^{2+\sigma}\right),
$$

where $P^{\star}=P+2 d\left(P, \mathcal{D}_{2}\right) \nu_{\bar{P}}$ and $\bar{P} \in \mathcal{D}_{2}$ is a unique point such that $d(P, \bar{P})=$ $2 d\left(P, \mathcal{D}_{2}\right)$, while $\sigma$ is a small positive number. Moreover, $v_{\bar{P}}$ is the outer unit normal at $\bar{P}$.

\section{Refinement of the projection}

Define

$H_{0}^{1}(\mathcal{D})=\left\{u \in H^{1}: u(x)=u(r, s), u=0\right.$ in $\mathcal{D}_{1}$ and $\mathcal{D}_{2} ; \frac{\partial u}{\partial v}=0$ in $\mathcal{D}_{3}$ and $\left.\mathcal{D}_{4}\right\}$

Define a norm on $H_{0}^{1}(\mathcal{D})$ as

$$
\|v\|_{\varepsilon}^{2}=\int_{\mathcal{D}} r^{M-1} r^{K-1}\left[\varepsilon^{2}|\nabla v|^{2} d x+v^{2}\right] d r d s .
$$

In this section we will refine the projection to incorporate the Neumann boundary condition on $\mathcal{D}_{3}$ and $\mathcal{D}_{4}$. We define a new projection as

$$
V_{\varepsilon, P}=\eta P U_{\varepsilon, P}
$$

where $0 \leq \eta \leq 1$ is smooth cut off function

$$
\eta(x)= \begin{cases}1 & \text { in } \mathcal{D} \cap B_{d / 2}(P) \\ 0 & \text { in } \mathcal{D} \backslash B_{d}(P)\end{cases}
$$

Here $d=\operatorname{dist}(P, \partial \mathcal{D})$ is dependent on $\varepsilon$. We will choose $d$ at the end of the proof.

We define

$$
u_{\varepsilon}=V_{\varepsilon, P}+\varphi_{\varepsilon, P} .
$$

Let $\varphi_{\varepsilon, P}=\varphi$. Using this ansatz, (1.3) reduces to

$$
\begin{cases}\varepsilon^{2} \Delta_{(r, s)} \varphi-\varphi+\varepsilon^{2} \frac{(M-1)}{r} \varphi_{r}+\varepsilon^{2} \frac{(K-1)}{s} \varphi_{r}+f^{\prime}\left(V_{\varepsilon, P}\right) \varphi=h & \text { in } \mathcal{D} \\ \varphi=0 & \text { on } \partial \mathcal{D}\end{cases}
$$

where $h=-S_{\varepsilon}\left[V_{\varepsilon, P_{\varepsilon}}\right]+N_{\varepsilon}[\varphi]$, while

$$
\begin{aligned}
S_{\varepsilon}\left[V_{\varepsilon, P}\right]= & \varepsilon^{2} \Delta_{(r, s)} V_{\varepsilon, P}+\varepsilon^{2} \frac{(M-1)}{r} V_{\varepsilon, P, r}+\varepsilon^{2} \frac{(K-1)}{s} V_{\varepsilon, P, s} \\
& -V_{\varepsilon, P}+f\left(V_{\varepsilon, P}\right)
\end{aligned}
$$


and

$$
N_{\varepsilon}\left[\varphi_{\varepsilon}\right]=\left\{f\left(V_{\varepsilon, P_{\varepsilon}}+\varphi\right)-f\left(V_{\varepsilon}\right)-f^{\prime}\left(V_{\varepsilon, P_{\varepsilon}}\right) \varphi\right\}
$$

Let

$$
E_{\varepsilon, P}=\left\{\omega \in H_{0}^{1}(\mathcal{D}),\left\langle\omega, \frac{\partial V_{\varepsilon, P}}{\partial r}\right\rangle_{\varepsilon}=\left\langle\omega, \frac{\partial V_{\varepsilon, P}}{\partial s}\right\rangle_{\varepsilon}=0\right\} .
$$

Let $\mathcal{D}_{\varepsilon}=\{z: \varepsilon z+P \in \mathcal{D}\}$.

Lemma 3.1. For any $z \in \mathcal{D}_{\varepsilon} \backslash B_{d / 2 \varepsilon}(P)$ we have

$$
P U_{\varepsilon, P}(z)=O\left(\varepsilon^{k}\right) \text {. }
$$

Proof. For any $z \in \mathcal{D}_{\varepsilon} \backslash B_{d / 2 \varepsilon}(P)$ we have

$$
\begin{aligned}
P U_{\varepsilon, P}(z) & \leq\left|U\left(\left|z-\frac{P}{\varepsilon}\right|\right)-v_{\varepsilon, P}(\varepsilon z)\right| \\
& =O\left(e^{-\left|x-\frac{P}{\varepsilon}\right|}+e^{-\left|x-\frac{P^{\star}}{\varepsilon}\right|}+\varepsilon^{2+\sigma}\right) \\
& =O\left(e^{-\frac{d\left(P, P^{\star}\right)}{\varepsilon}}+\varepsilon^{2+\sigma}\right) \\
& =O\left(e^{-\frac{2 d\left(P, \partial \mathcal{D}_{1}\right)}{\varepsilon}}+\varepsilon^{2+\sigma}\right)=O\left(\varepsilon^{k}\right) .
\end{aligned}
$$

Lemma 3.2. Let $P \in \Lambda_{\varepsilon, D}$. Then the energy expansion is given by

$$
\begin{aligned}
I_{\varepsilon}\left(V_{\varepsilon, P}\right) & =\int_{\mathcal{D}} r^{M-1} s^{K-1}\left(\frac{\varepsilon^{2}}{2}\left|\nabla V_{\varepsilon, P}\right|^{2}+\frac{1}{2} V_{\varepsilon, P}^{2}-\frac{1}{p+1} V_{\varepsilon, P}^{p+1}\right) d r d s \\
& =\gamma \varepsilon^{2} P_{1}^{M-1} P_{2}^{K-1}+\gamma_{1} \varepsilon^{2} P_{1}^{M-1} P_{2}^{K-1} U\left(\frac{2 d\left(P, \partial \mathcal{D}_{1}\right)}{\varepsilon}\right)+o\left(\varepsilon^{2}\right) U(k|\ln \varepsilon|)
\end{aligned}
$$

where $\gamma=\frac{p-1}{2(p+1)} \int_{\mathbb{R}^{2}} U^{p+1} d r d s$ and $\gamma_{1}=\frac{1}{2} \int_{\mathbb{R}^{2}} U^{p} e^{-r} d r d s$.

Proof. We obtain

$$
\begin{aligned}
I_{\varepsilon}\left(V_{\varepsilon, P}\right)= & \int_{\mathcal{D}} r^{M-1} s^{K-1}\left(\frac{\varepsilon^{2}}{2}\left|\nabla V_{\varepsilon, P}\right|^{2}+\frac{1}{2} V_{\varepsilon, P}^{2}-\frac{1}{p+1} V_{\varepsilon, P}^{p+1}\right) d r d s \\
= & \int_{\mathcal{D}} \eta^{2} r^{M-1}{ }_{s} K-1\left(\frac{\varepsilon^{2}}{2}\left|\nabla P U_{\varepsilon, P}\right|^{2}+\frac{1}{2} P U_{\varepsilon, P}^{2}-\frac{1}{p+1} P U_{\varepsilon, P}^{p+1}\right) d r d s \\
& +\frac{1}{p+1} \int_{\mathcal{D}} r^{M-1} s^{K-1}\left(\eta^{2}-\eta^{p+1}\right) P U_{\varepsilon, P}^{p+1} d r d s \\
& +\varepsilon^{2} \int_{\mathcal{D}} r^{M-1}{ }^{K-1} \eta \nabla \eta P U_{\varepsilon} \nabla P U_{\varepsilon} d r d s \\
& +\varepsilon^{2} \int_{\mathcal{D}} r^{M-1}{ }_{s} K-1|\nabla \eta|^{2}\left(P U_{\varepsilon, P}\right)^{2} d r d s \\
= & J_{1}+J_{2}+J_{3}+J_{4} .
\end{aligned}
$$


Hence we have

$$
\begin{aligned}
& J_{1}=\int_{\mathcal{D}} r^{M-1} s^{K-1}\left(\frac{\varepsilon^{2}}{2}\left|\nabla P U_{\varepsilon, P}\right|^{2}+\frac{1}{2} P U_{\varepsilon, P}^{2}-\frac{1}{p+1} P U_{\varepsilon, P}^{p+1}\right) d r d s \\
& -\int_{\mathcal{D}}\left(1-\eta^{2}\right) r^{M-1} s^{K-1}\left(\frac{\varepsilon^{2}}{2}\left|\nabla P U_{\varepsilon, P}\right|^{2}+\frac{1}{2} P U_{\varepsilon, P}^{2}-\frac{1}{p+1} P U_{\varepsilon, P}^{p+1}\right) d r d s \\
& =\int_{\mathcal{D}} r^{M-1} s^{K-1}\left(\frac{1}{2} U_{\varepsilon, P}^{p} P U_{\varepsilon, P}-\frac{1}{p+1} P U_{\varepsilon, P}^{p+1}\right) d r d s \\
& +\varepsilon^{2} \int_{\partial B_{d}(P)} r^{M-1} s^{K-1}\left(\frac{\partial P U_{\varepsilon, P}}{\partial r}+\frac{\partial P U_{\varepsilon, P}}{\partial s}\right) P U_{\varepsilon, P} d r d s \\
& -\varepsilon^{2} \int_{\partial B_{d / 2}(P)} r^{M-1} s^{K-1}\left(\frac{\partial P U_{\varepsilon, P}}{\partial r}+\frac{\partial P U_{\varepsilon, P}}{\partial s}\right) P U_{\varepsilon, P} d r d s \\
& =\varepsilon^{2}\left(\frac{1}{2}-\frac{1}{p+1}\right) \int_{\mathcal{D}_{\varepsilon}}\left(P_{1}+\varepsilon r\right)^{M-1}\left(P_{2}+\varepsilon s\right)^{K-1} U^{p+1}(z) d r d s \\
& +\frac{1}{2} \int_{\mathcal{D}} U_{\varepsilon, P}^{p} v_{\varepsilon} r^{M-1} s^{K-1} d r d s+O\left(\int_{\mathcal{D}} U_{\varepsilon, P}^{p-1} v_{\varepsilon}^{2} r^{M-1}{ }_{s}^{K-1} d r d s\right) \\
& +\varepsilon^{2} \int_{\partial B_{d}(P)} r^{M-1} s^{K-1}\left(\frac{\partial P U_{\varepsilon, P}}{\partial r}+\frac{\partial P U_{\varepsilon, P}}{\partial s}\right) P U_{\varepsilon, P} d r d s \\
& -\varepsilon^{2} \int_{\partial B_{d / 2}(P)} r^{M-1} s^{K-1}\left(\frac{\partial P U_{\varepsilon, P}}{\partial r}+\frac{\partial P U_{\varepsilon, P}}{\partial s}\right) P U_{\varepsilon, P} d r d s \\
& +\int_{\mathcal{D} \backslash B_{d / 2}} r^{M-1}{ }_{S} K-1\left(\frac{\varepsilon^{2}}{2}\left|\nabla P U_{\varepsilon, P}\right|^{2}+\frac{1}{2} P U_{\varepsilon, P}^{2}-\frac{1}{p+1} P U_{\varepsilon, P}^{p+1}\right) d r d s .
\end{aligned}
$$

Now we estimate

$$
\begin{aligned}
\varepsilon^{2}\left(\frac{1}{2}-\frac{1}{p+1}\right) \int_{\mathcal{D}_{\varepsilon}}\left(P_{1}+\varepsilon r\right)^{M-1}\left(P_{2}+\varepsilon s\right)^{K-1} U^{p+1}(z) d r d s \\
=\frac{p-1}{2(p+1)} \varepsilon^{2} P_{1}^{M-1} P_{2}^{K-1} \int_{\mathbb{R}^{2}} U^{p+1}(r, s) d r d s \\
\quad+o\left(\varepsilon^{2}\right) U(k|\ln \varepsilon|) .
\end{aligned}
$$


From Lemma 2.1, we compute the interaction term

$$
\begin{aligned}
\int_{\mathcal{D}} & U_{\varepsilon, P}^{p} v_{\varepsilon} r^{M-1} s^{K-1} d r d s \\
= & \varepsilon^{2} \int_{\mathcal{D}_{\varepsilon}} U^{p} U\left(\left|z-\frac{P-P^{\star}}{\varepsilon}\right|\right)\left(P_{1}+\varepsilon r\right)^{M-1}\left(P_{2}+\varepsilon s\right)^{K-1} d r d s \\
= & \varepsilon^{2} P_{1}^{M-1} P_{2}^{K-1} U\left(\left|\frac{P-P^{\star}}{\varepsilon}\right|\right)\left(\gamma_{1}+o(1)\right)+O\left(\varepsilon^{2+\sigma}\right) \\
= & \varepsilon^{2} P_{1}^{M-1} P_{2}^{K-1} U\left(\frac{2 d\left(P, \partial \mathcal{D}_{1}\right)}{\varepsilon}\right)\left(\gamma_{1}+o(1)\right)+O\left(\varepsilon^{2+\sigma}\right) \\
= & \varepsilon^{2} P_{1}^{M-1} P_{2}^{K-1} U\left(\frac{2 d\left(P, \partial \mathcal{D}_{1}\right)}{\varepsilon}\right)\left(\gamma_{1}+o(1)\right)+o\left(\varepsilon^{2}\right) U(k|\ln \varepsilon|) .
\end{aligned}
$$

Note that we have used the fact that $\frac{\left|P-P^{\star}\right|}{\varepsilon} \gg|z|$. Moreover, we obtain

$$
J_{2}=\int_{\mathcal{D}} r^{M-1}{ }_{s}{ }^{-1}\left(\eta^{2}-\eta^{p+1}\right) P U_{\varepsilon, P}^{p+1} d r d s=o\left(\varepsilon^{2}\right) U(k|\ln \varepsilon|) .
$$

Furthermore, we have

$$
\begin{gathered}
\varepsilon^{2} \int_{\partial B_{d}(P)} r^{M-1} s^{K-1}\left(\frac{\partial P U_{\varepsilon, P}}{\partial r}+\frac{\partial P U_{\varepsilon, P}}{\partial s}\right) P U_{\varepsilon, P} d r d s=o\left(\varepsilon^{2}\right) U(k|\ln \varepsilon|), \\
\varepsilon^{2} \int_{\partial B_{d / 2}(P)} r^{M-1} s^{K-1}\left(\frac{\partial P U_{\varepsilon, P}}{\partial r}+\frac{\partial P U_{\varepsilon, P}}{\partial s}\right) P U_{\varepsilon, P} d r d s=o\left(\varepsilon^{2}\right) U(k|\ln \varepsilon|), \\
J_{3}=\varepsilon^{2} \int_{\mathcal{D}} r^{M-1} s^{K-1} \eta \nabla \eta P U_{\varepsilon} \nabla P U_{\varepsilon} d r d s=o\left(\varepsilon^{2}\right) U(k|\ln \varepsilon|),
\end{gathered}
$$

and

$$
J_{4}=\varepsilon^{2} \int_{\mathcal{D}} r^{M-1} s^{K-1}|\nabla \eta|^{2}\left(P U_{\varepsilon, P}\right)^{2} d r d s=o\left(\varepsilon^{2}\right) U(k|\ln \varepsilon|) .
$$

Hence we obtain the result.

Remark 3.3. From lemma 3.2 we have

$$
\begin{aligned}
I_{\varepsilon}\left(V_{\varepsilon, P}\right) & =\int_{\mathcal{D}} r^{M-1} s^{K-1}\left(\frac{\varepsilon^{2}}{2}\left|\nabla V_{\varepsilon, P}\right|^{2}+\frac{1}{2} V_{\varepsilon, P}^{2}-\frac{1}{p+1} V_{\varepsilon, P}^{p+1}\right) d r d s \\
& =\gamma \varepsilon^{2} P_{1}^{M-1} P_{2}^{K-1}+\gamma_{1} \varepsilon^{2} P_{1}^{M-1} P_{2}^{K-1} U\left(\frac{2 d}{\varepsilon}\right)+o\left(\varepsilon^{2}\right) U(k|\ln \varepsilon|) .
\end{aligned}
$$


So if we expand the expression in $d$ and $\theta$ we have

$$
\begin{aligned}
\varepsilon^{-2} I_{\varepsilon}\left(V_{\varepsilon, P}\right)= & {\left[\gamma a^{M+K-2}+\gamma a^{M+K-3} d+\gamma_{1} a^{M+K-2} U\left(\frac{2 d}{\varepsilon}\right)\right] \cos ^{M-1} \theta \sin ^{K-1} \theta } \\
& +o\left(\varepsilon^{2}\right) U(k|\ln \varepsilon|)+O\left(\varepsilon^{2} d^{2}\right) .
\end{aligned}
$$

Note that the right-hand side is a function of $d$ and $\theta$ only and achieves its minimum in $d$ at a point $d \sim \varepsilon|\ln \varepsilon|$ provided $\cos ^{M-1} \theta \sin ^{K-1} \theta \neq 0$. This is the main reason of choosing the configuration space (2.14).

\section{The reduction}

In this section we will reduce the proof of Theorem 1.1 to finding a solution of the form $u_{\varepsilon}=V_{\varepsilon, P}+\varphi$ for (1.3) to a finite dimensional problem. We will prove that for each $P \in \Lambda_{\varepsilon, D}$, there is a unique $\varphi \in E_{\varepsilon, P}$ such that

$$
\left\langle I_{\varepsilon}^{\prime}\left(V_{\varepsilon, P}+\varphi\right), \eta\right\rangle_{\varepsilon}=0 \forall \eta \in E_{\varepsilon, P}
$$

Let

$$
J_{\varepsilon}(\varphi)=I_{\varepsilon}\left(V_{\varepsilon, P}+\varphi\right)
$$

We expand $J_{\varepsilon}(\varphi)$ near $\varphi_{\varepsilon, P}=0$ as

$$
J_{\varepsilon}(\varphi)=J_{\varepsilon}(0)+l_{\varepsilon, P}(\varphi)+\frac{1}{2} Q_{\varepsilon, P}(\varphi, \varphi)+R_{\varepsilon}(\varphi)
$$

where

$$
\begin{aligned}
l_{\varepsilon, P}(\varphi) & =\int_{\mathcal{D}} r^{M-1}{ }_{s} K-1\left[\varepsilon^{2} \nabla V_{\varepsilon, P} \nabla \varphi+V_{\varepsilon, P} \varphi-V_{\varepsilon, P}^{p} \varphi\right] d r d s \\
& =\int_{\mathcal{D}} r^{M-1} s^{K-1} S_{\varepsilon}\left[V_{\varepsilon, P}\right] \varphi d r d s \\
Q_{\varepsilon, P}(\varphi, \psi) & =\int_{\mathcal{D}} r^{M-1}{ }^{K-1}\left[\varepsilon^{2} \nabla \varphi \nabla \psi+\varphi \psi-p V_{\varepsilon, P}^{p-1} \varphi \psi\right] d r d s
\end{aligned}
$$

and

$$
\begin{aligned}
R_{\varepsilon}(\varphi)= & \frac{1}{p+1} \int_{\mathcal{D}} r^{M-1} s^{K-1}\left[\left(V_{\varepsilon, P}+\varphi\right)^{p+1}-\left(V_{\varepsilon, P}\right)^{p+1}\right. \\
& \left.-(p+1)\left(V_{\varepsilon, P}\right)^{p} \varphi-\frac{p(p+1)}{2}\left(V_{\varepsilon, P}\right)^{p-1} \varphi^{2}\right] d r d s
\end{aligned}
$$

We will prove in Lemma 4.1 that $l_{\varepsilon, P}(\varphi)$ is a bounded linear functional in $E_{\varepsilon, P}$. Hence by the Riesz representation theorem, there exists $l_{\varepsilon, P} \in E_{\varepsilon, P}$ such that

$$
\left\langle l_{\varepsilon, P}, \varphi\right\rangle_{\varepsilon}=l_{\varepsilon, P}(\varphi) \forall \varphi \in E_{\varepsilon, P} .
$$


In Lemma 4.2 we will prove that $Q_{\varepsilon, P}(\varphi, \eta)$ is a bounded linear operator from $E_{\varepsilon, P}$ to $E_{\varepsilon, P}$ such that

$$
\left\langle Q_{\varepsilon, P} \varphi, \eta\right\rangle_{\varepsilon}=Q_{\varepsilon, P}(\varphi, \eta) \forall \varphi, \eta \in E_{\varepsilon, P} .
$$

Thus finding a critical point of $J_{\varepsilon}(\varphi)$ is equivalent to solving the problem in $E_{\varepsilon, P}$ :

$$
l_{\varepsilon, P}+Q_{\varepsilon, P} \varphi+R_{\varepsilon}^{\prime}(\varphi)=0 .
$$

We will prove in Lemma 4.3 that the operator $Q_{\varepsilon, P}$ is invertible in $E_{\varepsilon, P}$. In Lemma 4.5, we will prove that, if $\varphi$ belongs to a suitable set, $R_{\varepsilon}^{\prime}(\varphi)$ is a small perturbation term in (4.4). Thus we can use the contraction mapping theorem to prove that (4.4) has a unique solution for each fixed $P \in \Lambda_{\varepsilon, D}$.

Lemma 4.1. The functional $l_{\varepsilon, P}: H_{0}^{1}(\mathcal{D}) \rightarrow \mathbb{R}$ defined in (4.1) is a bounded linear functional. Moreover, we have

$$
\left\|l_{\varepsilon, P}\right\|_{\varepsilon}=o(\varepsilon) \sqrt{U(k|\ln \varepsilon|)} .
$$

Proof. We have

$$
\begin{aligned}
& l_{\varepsilon, P}(\varphi)=\int_{\mathcal{D}} r^{M-1} s^{K-1} S_{\varepsilon}\left[V_{\varepsilon, P}\right] \varphi d r d s \\
& =\int_{\mathcal{D}} r^{M-1}{ }_{s} K-1\left[\varepsilon^{2} \Delta_{(r, s)} V_{\varepsilon, P}+\varepsilon^{2} \frac{(M-1)}{r} V_{\varepsilon, P, r}+\varepsilon^{2} \frac{(K-1)}{s} V_{\varepsilon, P, s}\right. \\
& \left.-V_{\varepsilon, P}+f\left(V_{\varepsilon, P}\right)\right] \varphi \\
& =\int_{\mathcal{D}} r^{M-1}{ }_{s} K-1\left[\varepsilon^{2} \Delta_{(r, s)} \eta P U_{\varepsilon, P}+\varepsilon^{2} \frac{(M-1)}{r}\left(\eta P U_{\varepsilon, P}\right)_{r}\right. \\
& \left.+\varepsilon^{2} \frac{(K-1)}{s}\left(\eta P U_{\varepsilon, P}\right)_{s}-\eta P U_{\varepsilon, P}+f\left(\eta P U_{\varepsilon, P}\right)\right] \varphi \\
& =\int_{\mathcal{D}} \eta r^{M-1}{ }_{s} K-1\left[\varepsilon^{2} \Delta_{(r, s)} P U_{\varepsilon, P}\right. \\
& \left.+\varepsilon^{2} \frac{(M-1)}{r} P U_{\varepsilon, P, r}+\varepsilon^{2} \frac{(K-1)}{s} P U_{\varepsilon, P, s}-P U_{\varepsilon, P}+f\left(P U_{\varepsilon, P}\right)\right] \varphi \\
& +\varepsilon^{2} \int_{\mathcal{D}} r^{M-1}{ }_{s}{ }^{K-1}\left[P U_{\varepsilon, P} \Delta_{(r, s)} \eta+\nabla P U_{\varepsilon, P} \nabla \eta\right] \varphi \\
& +\int_{\mathcal{D}} r^{M-1} s^{K-1}\left(\eta-\eta^{p}\right) P U_{\varepsilon, P}^{p} \varphi \\
& +\varepsilon^{2} \int_{\mathcal{D}} \eta r^{M-1}{ }_{s} K-1\left[\frac{(M-1)}{r} P U_{\varepsilon, P, r}+\frac{(K-1)}{s} P U_{\varepsilon, P, s}\right] \varphi \\
& +\varepsilon^{2} \int_{\mathcal{D}} r^{M-1} s^{K-1}\left[\eta_{r} \frac{(M-1)}{r} P U_{\varepsilon, P, r}+\eta_{s} \frac{(K-1)}{s} P U_{\varepsilon, P}\right] \varphi
\end{aligned}
$$




$$
\begin{aligned}
= & \int_{\mathcal{D}} \eta r^{M-1} s^{K-1}\left[f\left(P U_{\varepsilon, P}\right)-f\left(U_{\varepsilon, P}\right)\right] \varphi \\
& +\varepsilon^{2} \int_{\mathcal{D}} \eta r^{M-1} s^{K-1}\left[\frac{(M-1)}{r} P U_{\varepsilon, P, r}+\frac{(K-1)}{s} P U_{\varepsilon, P, s}\right] \varphi d r d s \\
& +\varepsilon^{2} \int_{\mathcal{D}} r^{M-1} s^{K-1}\left[\eta_{r} \frac{(M-1)}{r} P U_{\varepsilon, P, r}+\eta_{s} \frac{(K-1)}{s} P U_{\varepsilon, P}\right] \varphi \\
& +\int_{\mathcal{D}} r^{M-1}{ }^{K-1}\left(\eta-\eta^{p}\right) P U_{\varepsilon, P}^{p} \varphi d r d s \\
& +\varepsilon^{2} \int_{\mathcal{D}} r^{M-1}{ }^{K-1}\left[P U_{\varepsilon, P} \Delta_{(r, s)} \eta+\nabla P U_{\varepsilon, P} \nabla \eta\right] \varphi d r d s .
\end{aligned}
$$

Let

$$
\begin{aligned}
I_{1}= & \int_{\mathcal{D}} \eta r^{M-1} s^{K-1}\left[f\left(P U_{\varepsilon, P}\right)-f\left(U_{\varepsilon, P}\right)\right] \varphi d x \\
= & \int_{B_{d / 2}(P)} r^{M-1} s^{K-1}\left[f\left(P U_{\varepsilon, P}\right)-f\left(U_{\varepsilon, P}\right)\right] \varphi \\
& +\int_{B_{d} \backslash B_{d / 2}(P)} r^{M-1}{ }_{s}^{K-1}\left[f\left(P U_{\varepsilon, P}\right)-f\left(U_{\varepsilon, P}\right)\right] \varphi .
\end{aligned}
$$

Then using the decay estimates in (2.16), we obtain

$$
\begin{aligned}
I_{1} & \leq C \int_{B_{d}}\left(U_{\varepsilon, P}\right)^{p-1} v_{\varepsilon} \varphi d x \\
& \leq C \varepsilon \sqrt{U\left(\frac{P-P^{\star}}{\varepsilon}\right)}\left(\int_{\mathcal{D}}|\varphi|^{2} r^{M-1} s^{k-1} d r d s\right)^{\frac{1}{2}} \\
& =o(\varepsilon) \sqrt{U(k|\ln \varepsilon|)}\|\varphi\|_{\varepsilon} .
\end{aligned}
$$

Also it is easy to check that, all the other terms are of $o(\varepsilon) \sqrt{U(k|\ln \varepsilon|)}\|\varphi\|_{\varepsilon}$. Hence we obtain

$$
\left|l_{\varepsilon, P}(\varphi)\right|=o(\varepsilon) \sqrt{U(k|\ln \varepsilon|)}\|\varphi\|_{\varepsilon}
$$

and as a result

$$
\left\|l_{\varepsilon, P}\right\|_{\varepsilon}=o(\varepsilon) \sqrt{U(k|\ln \varepsilon|)} .
$$

Lemma 4.2. The bilinear form $Q_{\varepsilon, P}(\varphi, \eta)$ defined in (4.2) is a bounded linear. Furthermore,

$$
\left|Q_{\varepsilon, P}(\varphi, \eta)\right| \leq C\|\varphi\|_{\varepsilon}\|\eta\|_{\varepsilon}
$$

where $C$ is independent of $\varepsilon$. 
Proof. Using the Hölder's inequality, there exists $C>0$ such that

$$
\int_{\mathcal{D}} r^{M-1} s^{K-1} V_{\varepsilon, P}^{p-1} \varphi \eta d r d s \leq C \int_{\mathcal{D}} r^{M-1} s^{K-1}\left|\varphi\|\eta \mid \leq C\| \varphi\left\|_{\varepsilon}\right\| \eta \|_{\varepsilon}\right.
$$

and

$$
\left|\int_{\mathcal{D}} r^{M-1} s^{K-1}\left[\varepsilon^{2} \nabla \varphi \nabla \eta+\varphi \eta\right] d r d s\right| \leq C\|\varphi\|_{\varepsilon}\|\eta\|_{\varepsilon}
$$

Lemma 4.3. There exists $\rho>0$ independent of $\varepsilon$, such that

$$
\left\|Q_{\varepsilon, P} \varphi\right\|_{\varepsilon} \geq \rho\|\varphi\|_{\varepsilon} \forall \varphi \in E_{\varepsilon, P}, P \in \Lambda_{\varepsilon, P} .
$$

Proof. Suppose there exists a sequence $\varepsilon_{n} \rightarrow 0, \varphi_{n} \in E_{\varepsilon_{n}, P}, P \in \Lambda_{\varepsilon, D}$ such that $\left\|\varphi_{n}\right\|_{\varepsilon_{n}}=\varepsilon_{n}$ and

$$
\left\|Q_{\varepsilon_{n}} \varphi_{n}\right\|_{\varepsilon_{n}}=o\left(\varepsilon_{n}\right) .
$$

Let $\tilde{\varphi}_{n}(z)=\varphi_{n}\left(\varepsilon_{n} z+P\right)$ and $\mathcal{D}_{n}=\left\{y: \varepsilon_{n} z+P \in \mathcal{D}\right\}$ such that

$$
\int_{\mathcal{D}_{n}} r^{M-1} s^{K-1}\left[\left|\nabla \tilde{\varphi}_{n}\right|^{2}+\tilde{\varphi}_{n}^{2}\right]=\varepsilon_{n}^{-2} \int_{\mathcal{D}} r^{M-1} s^{K-1}\left[\varepsilon_{n}^{2}\left|\nabla \varphi_{n}\right|^{2}+\varphi_{n}^{2}\right]=1 .
$$

Hence there exists $\varphi \in H^{1}\left(\mathbb{R}^{2}\right)$ such that $\tilde{\varphi}_{n} \rightarrow \varphi \in H^{1}\left(\mathbb{R}^{2}\right)$ and hence $\tilde{\varphi}_{n} \rightarrow \varphi \in$ $L_{\text {loc }}^{2}\left(\mathbb{R}^{2}\right)$. We claim that

$$
\Delta_{(r, s)} \varphi-\varphi+p U^{p-1} \varphi=0 \text { in } \mathbb{R}^{2}
$$

that is, for all $\zeta \in C_{0}^{\infty}\left(\mathbb{R}^{2}\right)$,

$$
\int_{\mathbb{R}^{2}} r^{M-1} s^{K-1} \nabla \varphi \nabla \zeta+\int_{\mathbb{R}^{2}} r^{M-1} s^{K-1} \varphi \zeta=p \int_{\mathbb{R}^{2}} r^{M-1} s^{K-1} U^{p-1} \varphi \zeta
$$

Now

$$
\begin{aligned}
\int_{\mathcal{D}} r^{M-1} s^{K-1}\left[\varepsilon^{2} D \varphi_{n} D \zeta+\varphi_{n} \zeta-p V_{\varepsilon, P}^{p-1} \varphi_{n} \zeta\right] & =\left\langle Q_{\varepsilon_{n}, P} \varphi_{n}, \zeta\right\rangle_{\varepsilon} \\
& =o\left(\varepsilon_{n}\right)\|\zeta\|_{\varepsilon_{n}}
\end{aligned}
$$

which implies

$$
\int_{\mathcal{D}_{\varepsilon}} r^{M-1} s^{K-1}\left[\nabla \tilde{\varphi}_{n} \nabla \tilde{\zeta}+\tilde{\varphi}_{n} \tilde{\zeta}-p \tilde{V}_{\varepsilon, P}^{p-1} \tilde{\varphi}_{n} \tilde{\zeta}\right]=o(1)\|\tilde{\zeta}\|,
$$


where

$$
\begin{aligned}
\tilde{V}_{\varepsilon_{n}, P}(z) & =V_{\varepsilon_{n}, P_{n}}\left(\varepsilon_{n} z+P\right), \\
\tilde{P U_{\varepsilon_{n}, P}(z)} & =P U_{\varepsilon_{n}, P_{n}}\left(\varepsilon_{n} z+P\right), \\
\|\tilde{\zeta}\|^{2} & =\int_{\mathcal{D}_{n}} r^{M-1} s^{K-1}\left[|\nabla \tilde{\zeta}|^{2}+|\tilde{\zeta}|^{2}\right], \\
\tilde{E}_{\varepsilon_{n}, P} & =\left\{\tilde{\zeta}: \int_{\mathcal{D}_{n}} r^{M-1}{ }^{K-1} \nabla \tilde{\zeta} \nabla \tilde{W}_{n, r}+r^{M-1}{ }_{s} K-1 \tilde{\zeta} \tilde{W}_{n, r}\right. \\
& \left.=0=\int_{\mathcal{D}_{n}} r^{M-1}{ }^{K-1} \nabla \tilde{\zeta} \nabla \tilde{W}_{n, s}+r^{M-1}{ }_{s-1} \tilde{\zeta} \tilde{W}_{n, s}\right\},
\end{aligned}
$$

and $\tilde{W}_{n, r}=\varepsilon_{n} \frac{\partial V_{\varepsilon_{n}, P_{n}}\left(\varepsilon_{n} y+P_{n}\right)}{\partial r}, \tilde{W}_{n, s}=\varepsilon_{n} \frac{\partial V_{\varepsilon_{n}, P_{n}}\left(\varepsilon_{n} y+P_{n}\right)}{\partial s}$. Let $\zeta \in C_{0}^{\infty}\left(\mathbb{R}^{2}\right)$. Then we can choose $a_{1}, a_{2} \in \mathbb{R}$ such that

$$
\tilde{\zeta}_{n}=\zeta-\left[a_{1, n} \tilde{W}_{n, r}+a_{2, n} \tilde{W}_{n, s}\right]
$$

Note that $\tilde{W}_{n, r}$ satisfies the problem

$$
\begin{cases}-\Delta_{(r, s)} \tilde{W}_{n, r}+\tilde{W}_{n, r}=p \eta U^{p-1}(y) \frac{\partial U}{\partial r}+\Phi_{n}(y) & \text { in } \mathcal{D}_{n} \\ \tilde{W}_{n, r}=0 & \text { on } \partial \mathcal{D}_{n}\end{cases}
$$

where $\Phi_{n}(y)=\varepsilon_{n} \frac{\partial \eta}{\partial r}\left(U^{p}-\tilde{P} U_{\varepsilon, P}\right)+\varepsilon_{n} \frac{\partial}{\partial r}\left[2 \nabla_{(r, s)} \eta \nabla \tilde{P} U_{\varepsilon, P}+\Delta_{(r, s)} \eta \tilde{P} U_{\varepsilon, P}\right]$.

Then we claim that $\tilde{W}_{n, r}$ is bounded in $H_{0}^{1}\left(\mathcal{D}_{n}\right)$. Using the Hölder's inequality, we have

$$
\begin{aligned}
\int_{\mathcal{D}_{n}} r^{M-1} s^{N-1}\left[\left.\nabla \tilde{W}_{n, r}\right|^{2}+\tilde{W}_{n, r}^{2}\right]= & p \int_{\mathcal{D}_{n}} r^{M-1} s^{N-1} \eta U^{p-1} \frac{\partial U}{\partial r} \tilde{W}_{n, r} \\
& +\int_{\mathcal{D}_{n}} r^{M-1} s^{N-1} \Phi_{n} \tilde{W}_{n, r} \\
\leq & C\left(\int_{\mathcal{D}_{n}} r^{M-1} s^{k-1} \tilde{W}_{n, r}^{2}\right)^{\frac{1}{2}} \\
\leq & C\left(\int_{\mathcal{D}_{n}} r^{M-1} s^{N-1}\left[\left.\nabla \tilde{W}_{n, r}\right|^{2}+\tilde{W}_{n, r}^{2}\right]\right)^{\frac{1}{2}} .
\end{aligned}
$$

Hence $\int_{\mathcal{D}_{n}} r^{M-1} s^{N-1}\left[\left|\nabla \tilde{W}_{n, r}\right|^{2}+\tilde{W}_{n, r}^{2}\right]$ is uniformly bounded and as a result there exists $W_{r}$ such that

$$
\tilde{W}_{n, r} \rightarrow W_{r} \text { in } H^{1}\left(\mathbb{R}^{2}\right)
$$

up to a subsequence. Hence

$$
\tilde{W}_{n, r} \rightarrow W_{r} \text { in } L_{\text {loc }}^{2}
$$


Note that $W_{r}$ satisfies the problem,

$$
\left\{\begin{array}{l}
-\Delta_{(r, s)} W_{r}+W_{r}=p U^{p-1} \frac{\partial U}{\partial r} \quad \text { in } \mathbb{R}^{2} \\
\int_{\mathbb{R}^{2}} r^{M-1} s^{K-1}\left[\left|\nabla W_{r}\right|^{2}+\left|W_{r}\right|^{2}\right]=p \int_{\mathbb{R}^{2}} r^{M-1} s^{K-1} U^{p-1} \frac{\partial U}{\partial r} W_{r} .
\end{array}\right.
$$

We claim that $\tilde{W}_{n, r} \rightarrow W_{r}$ in $H^{1}\left(\mathbb{R}^{2}\right)$. First note that

$$
\begin{aligned}
\int_{\mathcal{D}_{n}} r^{M-1} s^{K-1}\left[\left|\nabla \tilde{W}_{n, r}\right|^{2}+\left|\tilde{W}_{n, r}\right|^{2}\right]= & p \int_{\mathcal{D}_{n}} r^{M-1} s^{K-1} U^{p-1} \frac{\partial U}{\partial r} \tilde{W}_{n, r} \\
& +\int_{\mathcal{D}_{n}} r^{M-1} s^{K-1} \Phi_{n} \tilde{W}_{n, r} \\
& \rightarrow p \int_{\mathbb{R}^{2}} r^{M-1} s^{K-1} U^{p-1} \frac{\partial U}{\partial r} W_{r} \\
= & \int_{\mathbb{R}^{2}} r^{M-1} s^{K-1}\left[\left|\nabla W_{r}\right|^{2}+\left|W_{r}\right|^{2}\right] d r d s .
\end{aligned}
$$

Here we have used that $\tilde{W}_{n, r}$ converges weakly in $L^{2}$. Hence $\tilde{W}_{n, r} \rightarrow W_{r}=\frac{\partial U}{\partial r}$ in $H^{1}$ strongly. Similarly, we can show that $\tilde{W}_{n, s} \rightarrow W_{s}=\frac{\partial U}{\partial s}$ in $H^{1}$ strongly. Now if we plug the value $\tilde{\zeta}_{n}$ in (4.7) and let $n \rightarrow \infty$, we obtain

$$
\begin{aligned}
& \int_{\mathbb{R}^{2}} r^{M-1} s^{K-1}\left[\nabla \varphi \nabla \zeta-p U^{p-1} \varphi \zeta+\varphi \zeta\right] \\
& =a_{1}\left(\int_{\mathbb{R}^{2}} r^{M-1} s^{K-1}\left[\nabla \varphi \nabla \frac{\partial U}{\partial r}+\varphi \frac{\partial U}{\partial r}-p U^{p-1} \varphi \frac{\partial U}{\partial r}\right]\right) \\
& \quad+a_{2}\left(\int_{\mathbb{R}^{2}} r^{M-1} s^{K-1}\left[\nabla \varphi \nabla \frac{\partial U}{\partial s}+\varphi \frac{\partial U}{\partial s}-p U^{p-1} \varphi \frac{\partial U}{\partial s}\right]\right)
\end{aligned}
$$

where $a_{i}=\lim _{n \rightarrow \infty} a_{i, n}$.

Using the non-degeneracy condition (2.7) we obtain

$$
\int_{\mathbb{R}^{N}} r^{M-1}{ }^{K-1}\left[\nabla \varphi \nabla \zeta+\varphi \zeta-p U^{p-1} \varphi \zeta\right]=0 .
$$

Hence we have (4.6).

Since $\varphi \in H^{1}\left(\mathbb{R}^{2}\right)$, it follows by non-degeneracy

$$
\varphi=b_{1} \frac{\partial U}{\partial r}+b_{2} \frac{\partial U}{\partial s} .
$$

Since $\tilde{\varphi}_{n} \in \tilde{E}_{\varepsilon_{n}, P}$, letting $n \rightarrow \infty$ in (4.7), we have

$$
\begin{aligned}
& \int_{\mathbb{R}^{2}} r^{M-1} s^{K-1} \nabla \varphi \nabla \frac{\partial U}{\partial r}=0 \\
& \int_{\mathbb{R}^{2}} r^{M-1} s^{K-1} \nabla \varphi \nabla \frac{\partial U}{\partial s}=0
\end{aligned}
$$


which implies $b_{1}=b_{2}=0$. Hence $\varphi=0$ and for any $R>0$ we have

$$
\int_{B_{\varepsilon_{n} R}(P)} r^{M-1} s^{K-1} \varphi_{n}^{2} d r d s=o\left(\varepsilon_{n}^{2}\right) .
$$

Hence

$$
\begin{aligned}
o\left(\varepsilon_{n}^{2}\right) & \geq\left\langle Q_{\varepsilon_{n}, P}\left(\varphi_{n}\right), \varphi_{n}\right\rangle_{\varepsilon_{n}} \geq\left\|\varphi_{n}\right\|_{\varepsilon_{n}}^{2}-p \int_{\mathcal{D}}\left(V_{\varepsilon_{n}, P}\right)^{p-1} \varphi_{n}^{2} \\
& \geq \varepsilon_{n}^{2}-o(1) \varepsilon_{n}^{2}
\end{aligned}
$$

which implies a contradiction.

Lemma 4.4. Let $R_{\varepsilon}(\varphi)$ be the functional defined by (4.3). Let $\varphi \in H_{0}^{1}(\mathcal{D})$, then

$$
\left|R_{\varepsilon}(\varphi)\right|=\varepsilon^{\tau}\|\varphi\|_{\varepsilon}^{2}
$$

and

$$
\left\|R_{\varepsilon}^{\prime}(\varphi)\right\|_{\varepsilon}=\varepsilon^{\tau}\|\varphi\|_{\varepsilon}
$$

for some $\tau>0$ small.

Proof. We have

$$
\begin{aligned}
\left|R_{\varepsilon}(\varphi)\right| & \leq o\left(\int_{\mathcal{D}} r^{M-1} s^{K-1} V_{\varepsilon, P}^{p-1} \varphi^{2}\right) \\
& \leq o(1) \int_{\mathcal{D}} r^{M-1} s^{K-1} V_{\varepsilon, P}^{p-1} \varphi^{2}=o(1)\|\varphi\|_{\varepsilon}^{2}
\end{aligned}
$$

Choosing $o(1)=\varepsilon^{\tau}$, we obtain the first estimate. The second estimate follows in a similar way.

Lemma 4.5. There exists $\varepsilon_{0}>0$ such that for $\varepsilon \in\left(0, \varepsilon_{0}\right]$, there exists a $C^{1}$ map $\varphi: E_{\varepsilon, P} \rightarrow H$, such that $\varphi \in E_{\varepsilon, P}$ we have

$$
\left\langle I_{\varepsilon}^{\prime}\left(V_{\varepsilon, P}+\varphi\right), \eta\right\rangle_{\varepsilon}=0, \quad \forall \eta \in E_{\varepsilon, P}
$$

Moreover, we have

$$
\|\varphi\|_{\varepsilon}=o(\varepsilon) \sqrt{U(k|\ln \varepsilon|)} .
$$

Proof. We have $l_{\varepsilon, P}+Q_{\varepsilon, P} \varphi+R_{\varepsilon}^{\prime}(\varphi)=0$. As $Q_{\varepsilon, P}^{-1}$ exists, the above equation is equivalent to solving

$$
Q_{\varepsilon, P}^{-1} l_{\varepsilon, P}+\varphi+Q_{\varepsilon, P}^{-1} R_{\varepsilon}^{\prime}(\varphi)=0
$$


Define

$$
\mathcal{G}(\varphi)=-Q_{\varepsilon, P}^{-1} l_{\varepsilon, P}-Q_{\varepsilon, P}^{-1} R_{\varepsilon}^{\prime}(\varphi) \quad \forall \varphi \in \Lambda_{\varepsilon, D}
$$

Hence the problem is reduced to finding a fixed point of the map $\mathcal{G}$.

For any $\varphi_{1} \in E_{\varepsilon, P}$ and $\varphi_{2} \in E_{\varepsilon, P}$ with $\left\|\varphi_{1}\right\|_{\varepsilon} \leq o\left(\varepsilon^{1-\tau}\right) \sqrt{U(k|\ln \varepsilon|)},\left\|\varphi_{2}\right\|_{\varepsilon} \leq$ $o\left(\varepsilon^{1-\tau}\right) \sqrt{U(k|\ln \varepsilon|)}$

$$
\left\|\mathcal{G}\left(\varphi_{1}\right)-\mathcal{G}\left(\varphi_{2}\right)\right\|_{\varepsilon} \leq C\left\|R_{\varepsilon}^{\prime}\left(\varphi_{1}\right)-R_{\varepsilon}^{\prime}\left(\varphi_{2}\right)\right\|_{\varepsilon} .
$$

From Lemma 4.4, we have

$$
\left\langle R_{\varepsilon}^{\prime}\left(\varphi_{1}\right)-R_{\varepsilon}^{\prime}\left(\varphi_{2}\right), \eta\right\rangle_{\varepsilon} \leq o(1)\left\|\varphi_{1}-\varphi_{2}\right\|_{\varepsilon}\|\eta\|_{\varepsilon} .
$$

Hence we have

$$
\left\|R_{\varepsilon}^{\prime}\left(\varphi_{1}\right)-R_{\varepsilon}^{\prime}\left(\varphi_{2}\right)\right\|_{\varepsilon} \leq o(1)\left\|\varphi_{1}-\varphi_{2}\right\|_{\varepsilon} .
$$

Hence $\mathcal{G}$ is a contraction as

$$
\left\|\mathcal{G}\left(\varphi_{1}\right)-\mathcal{G}\left(\varphi_{2}\right)\right\|_{\varepsilon} \leq \operatorname{Co}(1)\left\|\varphi_{1}-\varphi_{2}\right\|_{\varepsilon}
$$

Also for $\varphi \in E_{\varepsilon, P}$ with $\|\varphi\|_{\varepsilon} \leq o\left(\varepsilon^{1-\tau}\right) \sqrt{U(k|\ln \varepsilon|)}$, and $\tau>0$ sufficiently small

$$
\begin{aligned}
\|\mathcal{G}(\varphi)\|_{\varepsilon} & \leq C\left\|l_{\varepsilon, P}\right\|_{\varepsilon}+C\left\|R_{\varepsilon}^{\prime}(\varphi)\right\|_{\varepsilon} \\
& \leq C o(\varepsilon) \sqrt{U(k|\ln \varepsilon|)}+\operatorname{Co}\left(\varepsilon^{1-\tau+\tau}\right) \sqrt{U(k|\ln \varepsilon|)} \\
& \leq \operatorname{Co}(\varepsilon) \sqrt{U(k|\ln \varepsilon|)} .
\end{aligned}
$$

Hence

$$
\mathcal{G}: \Lambda_{\varepsilon, D} \cap B_{o\left(\varepsilon^{1-\tau}\right) \sqrt{U(k|\ln \varepsilon|)}}(0) \rightarrow \Lambda_{\varepsilon, D} \cap B_{o\left(\varepsilon^{1-\tau}\right) \sqrt{U(k|\ln \varepsilon|)}}(0)
$$

is a contraction map. Hence, by the contraction mapping principle there exists a unique $\varphi \in \Lambda_{\varepsilon, D} \cap B_{o\left(\varepsilon^{1-\tau}\right) \sqrt{U(k|\ln \varepsilon|)}}(0)$ such that $\varphi=\mathcal{G}(\varphi)$ and

$$
\|\varphi\|_{\varepsilon}=\|\mathcal{G}(\varphi)\|_{\varepsilon} \leq \operatorname{Co}(\varepsilon) \sqrt{U(k|\ln \varepsilon|)}
$$

We write $u_{\varepsilon}=V_{\varepsilon, P}+\varphi$. Then we have

$$
\begin{aligned}
I_{\varepsilon}\left(u_{\varepsilon}\right)= & I_{\varepsilon}\left(V_{\varepsilon, P}\right) \\
& +\int_{D} r^{M-1} s^{K-1}\left(\varepsilon^{2} \nabla V_{\varepsilon, P} \nabla \varphi-V_{\varepsilon, P} \varphi+f\left(V_{\varepsilon, P}\right) \varphi\right) d r d s \\
& +\frac{1}{2}\left(\int_{D} r^{M-1} s^{K-1}\left[\varepsilon^{2}|\nabla \varphi|^{2}-\varphi^{2}+f^{\prime}\left(V_{\varepsilon, P}\right) \varphi^{2}\right] d r d s\right) \\
& -\int_{D} r^{M-1} s^{K-1}\left[F\left(V_{\varepsilon, P}+\varphi\right)-F\left(V_{\varepsilon, P}\right)-\varepsilon f\left(V_{\varepsilon, P}\right) \varphi-\frac{1}{2} f^{\prime}\left(V_{\varepsilon, P}\right) \varphi^{2}\right] d r d s
\end{aligned}
$$


which can be expressed as

$$
\begin{aligned}
I_{\varepsilon}\left(u_{\varepsilon}\right)= & I_{\varepsilon}\left(V_{\varepsilon, P}\right) \\
& +\int_{\mathcal{D}} S_{\varepsilon}\left[V_{\varepsilon, P}\right] \varphi r^{M-1} s^{K-1} d r d s \\
& +\frac{1}{2}\left(\int_{\mathcal{D}}\left[\varepsilon^{2}|\nabla \varphi|^{2} d x-\varphi^{2}+f^{\prime}\left(V_{\varepsilon, P}\right) \varphi^{2}\right] r^{M-1} s^{K-1} d r d s\right) \\
& -\int_{\mathcal{D}} r^{M-1} s^{K-1}\left[F\left(V_{\varepsilon, P}+\varphi\right)-F\left(V_{\varepsilon, P}\right)-f\left(V_{\varepsilon, P}\right) \varphi-\frac{1}{2} f^{\prime}\left(V_{\varepsilon, P}\right) \varphi^{2}\right] d r d s \\
= & I_{\varepsilon}\left(V_{\varepsilon, P}\right)+O\left(\left\|l_{\varepsilon, P}\right\|_{\varepsilon}\|\varphi\|_{\varepsilon}+\|\varphi\|_{\varepsilon}^{2}+R_{\varepsilon}(\varphi)\right) \\
= & I_{\varepsilon}\left(V_{\varepsilon, P}\right)+o\left(\varepsilon^{2}\right) U(k|\ln \varepsilon|) .
\end{aligned}
$$

\section{The reduced problem: min-max procedure}

Proof of Theorem 1.1. Let $\mathcal{G}_{\varepsilon}(P)=\mathcal{G}_{\varepsilon}(d, \theta)=I_{\varepsilon}\left(u_{\varepsilon}\right)$. Consider the problem

$$
\min _{d \in \Lambda_{\varepsilon, P}} \max _{\theta_{0}-\delta \leq \theta \leq \theta_{0}+\delta} \mathcal{G}_{\varepsilon}(d, \theta) .
$$

To prove that $\mathcal{G}_{\varepsilon}(P)=I_{\varepsilon}\left(V_{\varepsilon, P}+\varphi\right)$ is a solution of (1.1), we need to prove that $P$ is a critical point of $\mathcal{G}_{\varepsilon}$, in other words we are required to show that $P$ is a interior point of $\Lambda_{\varepsilon, D}$.

For any $P \in \Lambda_{\varepsilon, P}$, from Lemma 4.3 we obtain

$$
\begin{aligned}
\mathcal{G}_{\varepsilon}(P)= & I_{\varepsilon}\left(V_{\varepsilon, P}\right)+O\left(\left\|l_{\varepsilon, P}\right\|_{\varepsilon}\|\varphi\|_{\varepsilon}+\|\varphi\|_{\varepsilon}^{2}+R_{\varepsilon}(\varphi)\right) \\
= & I_{\varepsilon}\left(V_{\varepsilon, P}\right)+o\left(\varepsilon^{2}\right) U(k|\ln \varepsilon|) \\
= & \varepsilon^{2} \gamma P_{1}^{M-1} P_{2}^{K-1}+\varepsilon^{2} \gamma_{1} P_{1}^{M-1} P_{2}^{K-1} U\left(\frac{2 d\left(P, \mathcal{D}_{1}\right)}{\varepsilon}\right) \\
& +o\left(\varepsilon^{2}\right) U(k|\ln \varepsilon|) .
\end{aligned}
$$

We have the expansion

$$
\begin{aligned}
\mathcal{G}_{\varepsilon}(d, \theta)=\gamma \varepsilon^{2}[ & a^{M+K-2}+a^{M+K-3} d+\gamma^{-1} \gamma_{1} a^{M+K-2} U\left(\frac{2 d\left(P, \mathcal{D}_{1}\right)}{\varepsilon}\right) \\
& \left.+O\left(d^{2}\right)\right] \cos ^{M-1} \theta \sin ^{K-1} \theta+o\left(\varepsilon^{2}\right) U(k|\ln \varepsilon|) \\
= & \gamma \varepsilon^{2}\left[a^{M+K-2}+a^{M+K-1} d+\gamma^{-1} \gamma_{1} a^{M+K-2} U\left(\frac{2 d}{\varepsilon}\right)\right] \\
& \times \cos ^{M-1} \theta \sin ^{K-1} \theta+o\left(\varepsilon^{2}\right) U(k|\ln \varepsilon|)+O\left(\varepsilon^{2} d^{2}\right) .
\end{aligned}
$$


It is clear that the maximum is attained at some interior point $\theta^{\prime} \in\left(\theta_{0}-\delta, \theta_{0}+\delta\right)$. Moreover, for this $\theta^{\prime}$, the minimum is attained at a interior point of $\Lambda_{\varepsilon, D}$. This finishes the proof.

\section{The reduced problem: max-max procedure}

Proof of Theorem 1.2. Here we obtain the critical point using a max-max procedure. The projection in the Neumann case is just $Q_{\varepsilon, P}$. Hence the reduced problem

$$
\begin{aligned}
\mathcal{R}_{\varepsilon}(P)= & \varepsilon^{2} \gamma P_{1}^{M-1} P_{2}^{K-1}-\varepsilon^{2} \gamma_{1} P_{1}^{M-1} P_{2}^{K-1} U\left(\frac{2 d\left(P, \mathcal{D}_{2}\right)}{\varepsilon}\right) \\
& +o\left(\varepsilon^{2}\right) U(k|\ln \varepsilon|) .
\end{aligned}
$$

Consider

$$
\max _{d \in \Lambda_{\varepsilon, N}} \max _{\theta_{0}-\delta \leq \theta \leq \theta_{0}+\delta} \mathcal{R}_{\varepsilon}(d, \theta) .
$$

We have the expansion

$$
\begin{aligned}
\mathcal{R}_{\varepsilon}(d, \theta)=\gamma \varepsilon^{2}\left[b^{M+K-2}-b^{M+K-3} d-\gamma^{-1} \gamma_{1} b^{M+K-2} U\left(\frac{2 d\left(P, \mathcal{D}_{2}\right)}{\varepsilon}\right)\right. & \left.\quad+O\left(d^{2}\right)\right] \cos ^{M-1} \theta \sin ^{K-1} \theta+o\left(\varepsilon^{2}\right) U(k|\ln \varepsilon|) \\
= & \gamma \varepsilon^{2}\left[b^{M+K-2}-b^{M+K-3} d-\gamma^{-1} \gamma_{1} b^{M+K-2} U\left(\frac{2 d}{\varepsilon}\right)\right] \\
& \times \cos ^{M-1} \theta \sin ^{K-1} \theta+o\left(\varepsilon^{2}\right) U(k|\ln \varepsilon|)+O\left(\varepsilon^{2} d^{2}\right) .
\end{aligned}
$$

It is clear that the maximum in $\theta$ is attained at some interior point $\theta^{\prime} \in\left(\theta_{0}-\delta, \theta_{0}+\right.$ $\delta)$. Moreover, for this $\theta^{\prime}$, the maximum is attained at a interior point $d$ of $\Lambda_{\varepsilon, N}$ if we choose $k>0$ to be sufficiently small. Hence Theorem 1.2 is proved.

\section{References}

[1] A. Ambrosetti, A. Malchiodi and W. Ni, Singularly perturbed elliptic equations with symmetry: existence of solutions concentrating on spheres. I, Comm. Math. Phys. 235 (2003), 427-466.

[2] A. Ambrosetti, A. Malchiodi and W. Ni, Singularly perturbed elliptic equations with symmetry: existence of solutions concentrating on spheres. II, Indiana Univ. Math. J. 53 (2004), 297-329.

[3] M. Del PINO and P. Felmer, Spike-layered solutions of singularly perturbed elliptic problems in a degenerate setting, Indiana Univ. Math. J. 48 (1999), 883-898.

[4] M. Del PINO, P. Felmer and J. WeI, On the role of mean curvature in some singularly perturbed Neumann problems, SIAM J. Math. Anal. 31 (1999), 63-79. 
[5] M. Del Pino, M. Kowalczyk and J. Wei, Concentraton on curves for nonlinear Schrödinger equations, Comm. Pure Appl. Math. 60 (2007), 113-146.

[6] B. GidAS, W. Ni and L. NiRENBERG, Symmetry and related properties via the maximum principle, Comm. Math. Phys. 68 (1979), 209-243.

[7] P. Esposito, G. Mancini, S. S ANTRA and P. SRikanth, Asymptotic behavior of radial solutions for a semilinear elliptic problem on an annulus through Morse index, J. Differential Equations 239 (2007), 1-15.

[8] C. GUI, Multi-peak solutions for a semilinear Neumann problem, Duke Math. J. 84 (1996), 739-769.

[9] F. LIN, W. M. NI and J. WEI, On the number of interior peak solutions for a singularly perturbed Neumann problem, Comm. Pure Appl. Math. 60 (2007), 252-281.

[10] Y. Y. LI, On a singularly perturbed equation with Neumann boundary condition, Comm. Partial Differential Equations 23 (1998), 487-545.

[11] A. MALChIODI and M. Montenegro, Boundary concentration phenomena for a singularly perturbed elliptic problem, Comm. Pure Appl. Math. 55 (2002), 1507-1568.

[12] A. MALCHIODI and M. MONTENEGRO, Multidimensional boundary layers for a singularly perturbed Neumann problem, Duke Math. J. 124 (2004), 105-143.

[13] F. PACELla and P. N. SRIKANTH, A reduction method for semilinear elliptic equations and solutions concentrating on sphere, J. Funct. Anal. 266 (2014), 6456-6472.

[14] B. RUF and P. SRIKANTH, Singularly perturbed elliptic equations with solutions concentrating on a 1-dimensional orbit, J. Eur. Math. Soc. (JEMS) 12 (2010), 413-427.

[15] B. RUF and P. SRIKANTH, Concentration on Hopf-fibres for singularly perturbed elliptic equations, J. Funct. Anal. 267 (2014), 2353-2370.

[16] W.-M. NI, Qualitative properties of solutions to elliptic problems, In: "Stationary Partial Differential Equations", Vol. I, Handb. Differential Equations, North-Holland, Amsterdam, 2004, 157-233.

[17] W.-M. NI and I. TAKAGI, On the shape of least-energy solutions to a semilinear Neumann problem, Comm. Pure Appl. Math. 4 (1991), 819-851.

[18] W. M. NI and I. TAKAGI, Locating the peaks of least-energy solutions to a semilinear Neumann problem, Duke Math. J. 70 (1993), 247-281.

[19] W. M. NI and J. WEI, On the location and profile of spike-layer solutions to singularly perturbed semilinear Dirichlet problems, Comm. Pure Appl. Math. 48 (1995), 731-768.

[20] W. M. NI and J. WEI, Diffusion, cross-diffusion, and their spike-layer steady states, Not. Amer. Math. Soc. 45 (1998), 9-18.

[21] J. WEI, On the boundary spike layer solutions of singularly perturbed semilinear Neumann problem, J. Differential Equations 134 (1997), 104-133.

[22] J. WEI and M. WINTER, Multiple boundary spike solutions for a wide class of singular perturbation problems, J. London Math. Soc. 59 (1999), 585-606.

Department of Basic Mathematics

Centro de Investigacióne en Mathematicás

Guanajuato, México

sanjiban@cimat.mx

Department of Mathematics

The University of British Columbia

Vancouver, Canada

jcwei@math.ubc.ca 